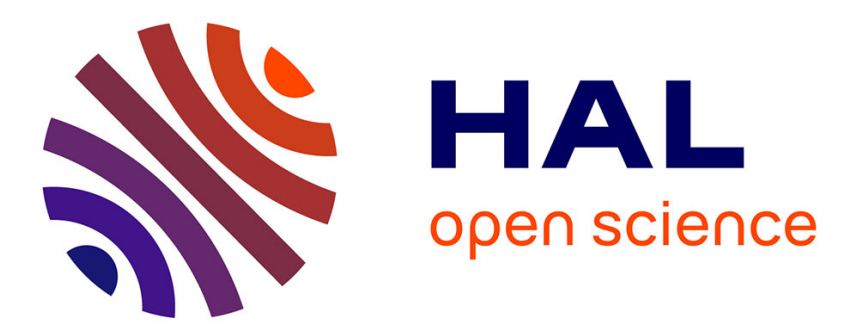

\title{
Étude comparative tensiomètre automatique versus doppler à ultrasons dans la mesure de l'index de pression systolique à la cheville (Ipsc)
}

\author{
Jean-François Hamel, M. Tanguy, David Foucaud, Serge Fanello
}

\section{- To cite this version:}

Jean-François Hamel, M. Tanguy, David Foucaud, Serge Fanello. Étude comparative tensiomètre automatique versus doppler à ultrasons dans la mesure de l'index de pression systolique à la cheville (Ipsc). Journal des Maladies Vasculaires, 2010, 35 (3), pp.169-174. 10.1016/j.jmv.2010.03.001 . hal03389587

\section{HAL Id: hal-03389587 \\ https://univ-angers.hal.science/hal-03389587}

Submitted on 21 Oct 2021

HAL is a multi-disciplinary open access archive for the deposit and dissemination of scientific research documents, whether they are published or not. The documents may come from teaching and research institutions in France or abroad, or from public or private research centers.
L'archive ouverte pluridisciplinaire $\mathbf{H A L}$, est destinée au dépôt et à la diffusion de documents scientifiques de niveau recherche, publiés ou non, émanant des établissements d'enseignement et de recherche français ou étrangers, des laboratoires publics ou privés. 
Disponible en ligne sur

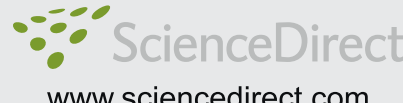

www.sciencedirect.com
Elsevier Masson France

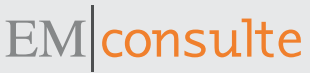

www.em-consulte.com

ARTICLE ORIGINAL

\title{
Étude comparative tensiomètre automatique versus doppler à ultrasons dans la mesure de l'index de pression systolique à la cheville (Ipsc)
}

\section{Comparison of the automated oscillometric method with Doppler ultrasound method to access the Ankle-Brachial Pressure Index (ABPI)}

\author{
J.-F. Hamel, M. Tanguy, D. Foucaud, S. Fanello* \\ Departement universitaire de santé publique, CHU d'Angers, 4, rue Larrey, 49933 Angers cedex 09, France
}

Reçu le 14 janvier 2010 ; accepté le $1^{\text {er }}$ mars 2010

Disponible sur Internet le 2 avril 2010

\author{
MOTS CLÉS \\ AOMI ; \\ IPSC ; \\ Doppler ; \\ Tensiomètre \\ automatique ; \\ Étude comparative
}

\begin{abstract}
Résumé
Objectif. - L'évaluation de l'index de pression systolique à la cheville (IPSC) est la méthode non invasive la plus simple de dépistage de l'artériopathie des membres inférieurs (AOMI) mais reste peu usitée en pratique courante, car elle est chronophage, implique un équipement et une formation appropriés. Le but de notre étude est d'évaluer la validité et la fiabilité de la mesure de L'IPSC à l'aide d'un tensiomètre automatique par comparaison avec les résultats obtenus par doppler continu, méthode de référence.

Méthodes. - Les patients de 65 ans et plus hospitalisés au CHU d'Angers de juillet 2008 à mars 2009 en médecine interne et n'ayant pas d'AOMI documentée au moment de l'inclusion ont participé à l'étude. Les critères d'exclusion étaient la présence d'une fibrillation auriculaire et une incompressibilité des artères distales des membres inférieurs. Pour chaque patient, l'IPSC a été évalué par tensiomètre automatique et par la méthode Doppler de référence; ces mesures étant faites par le même examinateur. La concordance entre les deux mesures était appréciée par la méthode de Bland et Altman et le coefficient de corrélation intraclasse (ICC).

Résultats. - Sur 287 patients participant à l'étude, 221 ont étés inclus. Cinquante-six (25\%) de ces patients avaient un IPSC < 0,90. La concordance entre les deux méthodes était médiocre: ICC à 0,35 $\pm 0,08$ et moyenne à $0,08 \pm 0,26$ selon la méthode de Bland et Altman.

Conclusion. - La méthode de dépistage de l'AOMI par mesure de l'IPSC par tensiomètre automatique ne peut être retenue comme une alternative à la méthode Doppler.

(c) 2010 Elsevier Masson SAS. Tous droits réservés.
\end{abstract}

\footnotetext{
* Auteur correspondant.

Adresse e-mail : sefanello@chu-angers.fr (S. Fanello).
} 


\section{KEYWORDS}

Peripheral artery

disease;

ABPI;

Doppler;

Automatic blood

pressure device;

Comparative study

\section{Summary}

Objective. - The Ankle-Brachial Pressure Index (ABPI) is the easiest non-invasive method for the assessment of peripheral arterial diseases (PAD) but remains uncommon in general practice, because of the need for training and specific devices. The purpose of this survey was to assess the reliability of the automated oscillometric measurement of the ABPI, compared with the gold-standard Doppler ultrasound measurement.

Methods. - A study was conducted on patients aged 65 years and over without diagnosed PAD hospitalized in the Teaching Hospital of Angers (France) from July 2008 to March 2009. Incompressive ankle arteries and atrial fibrillation were exclusion criteria. ABPI measurements were performed by the same examiner with oscillometric and Doppler devices. Reproducibility was assessed by the intra-class correlation coefficient of agreement (ICC) and the Bland-Altman method.

Results. - Out of the 287 eligible hospitalized patients, 221 patients were included in the study and $56(25 \%)$ had an ABPI below 0.90. The inter-method reliability is poor (ICC $=0.35 \pm 0.08$ ) and the mean difference between the methods was $0.08 \pm 0.26$ according to the Bland-Altman method.

Conclusion. - Automatic oscillometric devices cannot be recommended as a reliable alternative to access the ABPI.

(c) 2010 Elsevier Masson SAS. All rights reserved.

\section{Introduction}

Les pathologies cardiovasculaires sont une des premières causes de décès dans les pays développés [1,2]. Avec l'accroissement de l'espérance de vie, on s'attend à une progression de la prévalence des maladies cardiovasculaires.

Les sujets porteurs d'artériopathie des membres inférieurs $(\mathrm{AOMI})$ - même au stade infraclinique - sont à haut risque cardiovasculaire en raison du risque accru de développer une athérosclérose étendue à d'autres territoires vasculaires (coronaires, carotides, artères cérébrales) $[3,4]$ ainsi que des répercussions cardiovasculaires, tels l'infarctus du myocarde et la mort subite [5-7]. Par conséquent, le dépistage de l'artériopathie a un impact sur la démarche de soins $[8,9]$.

L'évaluation de l'index de pression systolique à la cheville (IPSC) est la méthode non invasive la plus simple de dépistage de l'AOMI [10] ; elle est sensible $(90 \%)$ et très spécifique ( $98 \%)$ dans la détection des sténoses de $50 \%$ et plus des artères des membres inférieurs [11]. Les autres méthodes cliniques de dépistage de l'AOMI ne sont pas aussi efficaces; ainsi l'absence de pouls distaux n'implique pas une artériopathie sous-jacente [12] alors que des pouls peuvent être perçus chez un artériopathe [13].

Ce mode simple de détection reste peu usité en pratique courante, car il est chronophage [14] et implique un équipement spécifique et une formation $[15,16]$. Un test automatisé et simple à réaliser pourrait améliorer la mesure de l'IPSC et par-là le dépistage d'AOMI.

Bien que la détermination de la tension artérielle par tensiomètre automatique soit approuvée pour la mesure de la pression humérale et reconnue comme généralement disponible, fiable et simple à mettre en œuvre $[17,18]$, son usage n'est pas pleinement validé dans le calcul de l'IPSC, malgré les avantages pratiques qui pourraient en résulter (méthode plus rapide et moins opérateur-dépendante).

Le premier article à faire état de l'utilisation d'un tensiomètre automatique pour évaluer la pression artérielle au membre inférieur fut publié en 1987 [19]. Depuis, plusieurs études ont traité de la fiabilité de la mesure de l'IPSC par tensiomètre automatique [20-26]. Dans leur ensemble, elles ont retrouvé une bonne corrélation entre l'IPSC mesuré par le tensiomètre automatique et celui donné par le Doppler à ultrasons. Cependant, dans ces articles les auteurs se sont servis d'outils statistiques comme les coefficients de corrélation Pearson et Spearman, la régression linéaire ou la comparaison de moyennes pour juger de la concordance entre ces deux méthodes. Or ces techniques statistiques testent non pas la concordance entre les variables étudiées, mais leur degré de «liaison» [27].

Tester la concordance entre deux séries d'observations impose donc d'utiliser d'autres méthodes statistiques. La méthode d'analyse de concordance de deux séries de valeurs consiste à étudier l'écart existant au sein des couples de valeurs de la même grandeur obtenus par deux méthodes différentes. Cette méthode est l'outil adapté pour l'appréciation clinique d'une nouvelle technique de mesure par rapport à une technique utilisée en référence. Il existe essentiellement deux méthodes permettant d'évaluer la concordance de variables quantitatives: l'analyse de la reproductibilité par la méthode de Bland et Altman [28] et le coefficient de corrélation intraclasse (ICC) qui correspond à un modèle d'analyse de la variance [29].

À notre connaissance, une seule étude a mesuré la concordance entre les deux méthodes de mesure de l'IPSC par le coefficient de corrélation intraclasse (ICC) [30]. Cette étude a retrouvé une fiabilité modérée avec un ICC $=0,62$ (intervalle de confiance à $95 \%$ : $[0,41-0,76]$ ) et en a déduit une mauvaise concordance entre la méthode Doppler et celle utilisant un tensiomètre automatique; l'étude portait sur 78 personnes.

Le but de notre étude est d'évaluer la validité et la fiabilité de la méthode de calcul de l'IPSC par tensiomètre automatique comparativement à celle de référence par doppler continu dans une étude clinique réalisée sur une plus large population de patients. 


\section{Population et méthode}

L'IPSC est le rapport de la pression systolique à la cheville sur la pression systolique humérale. Pour chaque jambe, la plus élevée des deux pressions mesurées au pouls pédieux et au pouls tibial postérieur est utilisée comme numérateur de L'IPSC, et la plus élevée des deux pressions humérales comme dénominateur. L'auscultation artérielle assurée grâce à un capteur doppler placé sur une artère distale permet de mesurer les pressions humérales bilatérales ainsi que les pressions systoliques aux chevilles. L'index de pression systolique à la cheville est le paramètre clé du diagnostic précoce et de la surveillance des patients suspects d'AOMI [31]. La valeur seuil pathologique pour le diagnostic d'AOMl est 0,90. En dessous, le diagnostic est retenu et constitue la définition de l'AOMI. La méthode de référence utilisait le doppler manuel (cf. procédure).

\section{Critères d'inclusion}

Tous les patients de 65 ans et plus, hospitalisés au centre hospitalier universitaire (CHU) d'Angers entre juillet 2008 et mars 2009 dans le service de médecine interne et n'ayant pas d'AOMI documentée étaient éligibles. Les patients aux artères distales des membres inférieurs incompressibles (IPSC Doppler $>1,30$ ) et/ou présentant une fibrillation auriculaire étaient exclus de l'analyse, par manque de fiabilité des mesures par brassard automatique dans cette population de patients [32].

\section{Procédure}

Les informations démographiques ont été collectées une fois le consentement des patients recueillis.

Les patients étaient installés en décubitus pendant dix à 15 minutes avant les prises de pressions. La mesure de la pression artérielle par méthode oscillométrique a été réalisée à partir d'un tensiomètre automatique (Omron M6 HEM-7001-E with Intellisense ${ }^{T M}$ ), validé cliniquement par le protocole international de la société européenne d'hypertension (ESH) [33]. Ce modèle a une précision évaluée à $2 \pm 3,3 \mathrm{mmHg}$ [34]. Le brassard était gonflé à des valeurs suprasystoliques (abolition du flux artériel), puis dégonflé. Le capteur inclus dans le brassard détectait les variations de pression induites par les pulsations artérielles pendant sa déflation.

Pour la méthode Doppler, on employait un appareil manuel à fréquence de sonde $8 \mathrm{MHz}$ (MINI DOPPLEX Nondirectional Doppler D900, Huntleigh Technology, Luton, UK). On auscultait les artères humérales, tibiales postérieures et pédieuses avec la sonde jusqu'à la levée de la compression et l'apparition du premier flux systolique audible sur l'axe étudié.

Toutes les mesures ont été effectuées par le même examinateur dans l'optique d'éliminer des variations interopérateur.

\section{Analyse des données}

Les IPSC obtenus par les deux méthodes ont été comparés. Les sensibilités, spécificités, valeurs prédictives positives et négatives de la méthode par tensiomètre automatique pour dépister un IPSC anormal ont été déterminées en prenant le calcul d'IPSC par mesure doppler comme référence.

\section{Nombre de sujets nécessaires}

Le critère de jugement principal était la mesure de l'IPSC par tensiomètre automatique. Il est à comparer avec l'IPSC évalué par stylo-Doppler. On définit à l'avance un risque de première espèce $\alpha$ fixé à $5 \%$. Le nombre de sujets nécessaires a été calculé à l'aide de la formule $n=\left(\varepsilon^{2} p q\right) / i^{2}$; où $\varepsilon$ représente l'écart réduit correspondant au risque $\alpha$ dans la table de l'écart réduit et $i$ représente l'intervalle de confiance choisi.

Une étude [35] réalisée sur 101 sujets dénombrait 25 patients dont l'IPSC était inférieur à 0,9 . À la suite d'une échographie Doppler de contrôle, le diagnostic d'AOMI était confirmé dans 18 cas sur 25 (72\%). Dans le cadre de notre étude, un intervalle de confiance de la valeur prédictive positive de $\pm 0,12$ a été choisi. Le nombre de sujets nécessaire ayant un IPSC inférieur à 0,9 était de 54 et notre étude nécessitait un effectif total de 216 patients.

La comparaison de deux mesures selon la méthode de Bland et Altman consistait à étudier l'écart observé au sein des couples de valeurs de l'IPSC obtenues par tensiomètre automatique et par Doppler et d'en déduire, sur l'ensemble de la population observée, le biais, la précision et les limites de l'intervalle de confiance à $95 \%$ qui permettront de statuer sur la concordance des deux séries de valeurs. Cette méthode est un outil adapté pour l'appréciation clinique d'une nouvelle technique de mesure par rapport à une technique utilisée en référence [36].

La saisie des données a été réalisée grâce au tableur Excel $^{\odot}$ et l'analyse statistique effectuée avec le logiciel SPSS $^{\odot}$ version 16.0 pour Windows ${ }^{\odot}$.

300 patients hospitalisés au C.H.U. d'Angers de juillet 2008 à mars 2009 éligibles

\section{CONSENTEMENT}

287 patients participent à l'étude

(146 femmes et 141 hommes)

$$
\begin{aligned}
\Rightarrow & \text { CRITERES D'EXCLUSION } \\
& -45 \text { patients pour Fibrillation Auriculaire } \\
& -21 \text { pour Médiacalcose }
\end{aligned}
$$

221 patients inclus

l'IPSC est évalué par le même examinateur

○ par tensiomètre automatique

- par la méthode doppler de référence

Schéma 1 Design de l'étude. Study set-up. 


\section{Résultats}

\section{Données générales (Schéma 1)}

Sur les 300 patients éligibles, 287 ont accepté de participer à l'étude. L'âge moyen des patients était de $80,5 \pm 15,6$ ans ; il y avait 146 femmes (51\%) pour 141 hommes. Quarante-cinq patients $(16 \%)$ qui souffraient de fibrillation auriculaire ont été exclus ainsi que 21 autres $(9 \%)$ en raison d'une incompressibilité des artères distales des membres inférieurs.

Sur les 221 sujets restants, $56(25,3 \%)$ avaient un IPSC $<0,9$ (Fig. 1). Cinq patients (2,3\%) présentaient des symptômes aux membres inférieurs sans diagnostic d'AOMI connu.

La prévalence des risques cardiovasculaires s'établissait comme suit ; tabagisme: $6,3 \%$, diabète : $16,7 \%$, hypertension : $58,4 \%$, hypercholestérolémie : $31,2 \%$, obésité : $14,0 \%$. On comptait respectivement $11,8 \%$ et $11,3 \%$ d'antécédents de coronaropathie et d'accident cérébrovasculaire.

\section{Fiabilité de la mesure de I'IPSC par tensiomètre automatique}

Il est apparu une différence significative entre la mesure de I'IPSC par tensiomètre automatique et celle par Doppler: la différence des moyennes entre les deux méthodes était de $0,08(D S=0,14)$ avec $p<10^{-4}$. La différence de résultats entre les deux méthodes de mesure suivait une distribution normale avec une différence non significative $(p=0,056)$ selon le test de Kolmogorov-Smirnov.

La concordance entre les deux méthodes était mauvaise : le coefficient de corrélation intraclasse (ICC) était à 0,35 avec un intervalle de confiance (IC) à $95 \%=[0,27-0,42]$ (Fig. 2). La méthode de Bland et Altman illustre la différence

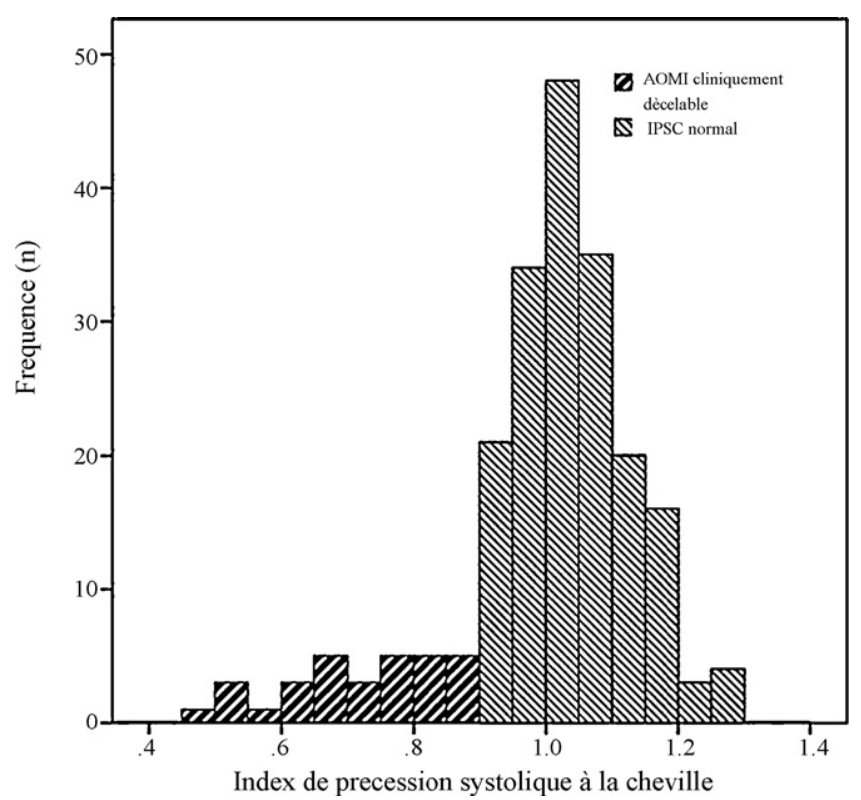

Figure 1 Distribution de l'IPSC : 25,3\% des patients présentaient un IPSC pathologique.

Bar chart of ABPI distribution: $25.3 \%$ of patients had an abnormal ABPI.

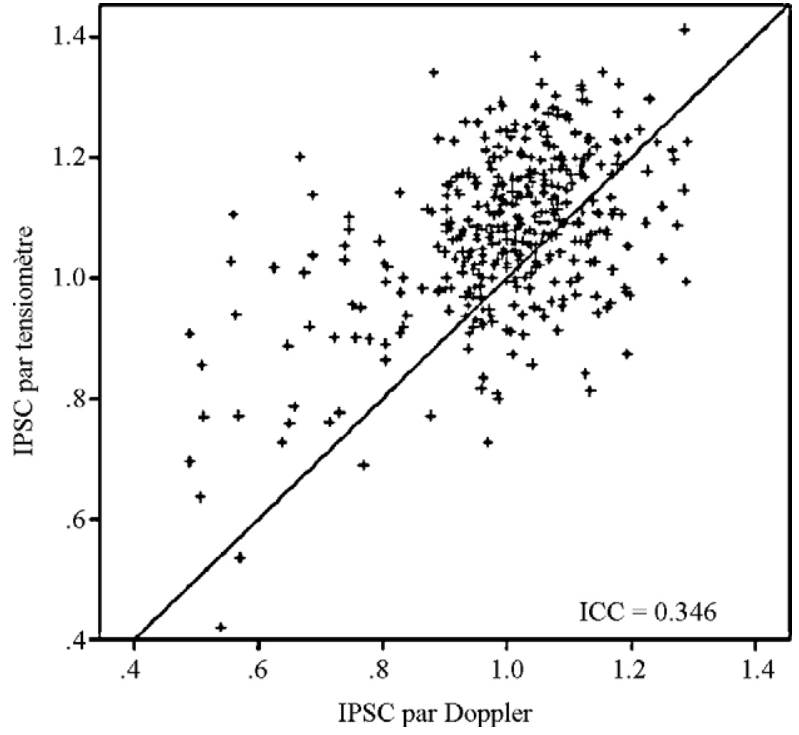

Figure 2 Diagramme de dispersion de la mesure de l'IPSC par tensiomètre automatique et par Doppler. L'ICC entre les deux méthodes était de 0,346 . IC $95 \%=[0,268-0,420]$.

Scatter plot of ABPI measurments: automated oscillometric method versus Doppler. The ICC between the two methods was 0.346 [95\% Cl: 0.268-0.420].

individuelle entre les deux méthodes : moyenne à 0,08 (IC à $95 \%=[-0,18-0,35])$ (Fig. 3).

Comparé à la méthode de référence (Doppler à ultrasons), le dépistage d'une AOMI par tensiomètre automatique avait une sensibilité de $35 \pm 6 \%$, une spécificité de $96 \pm 3 \%$

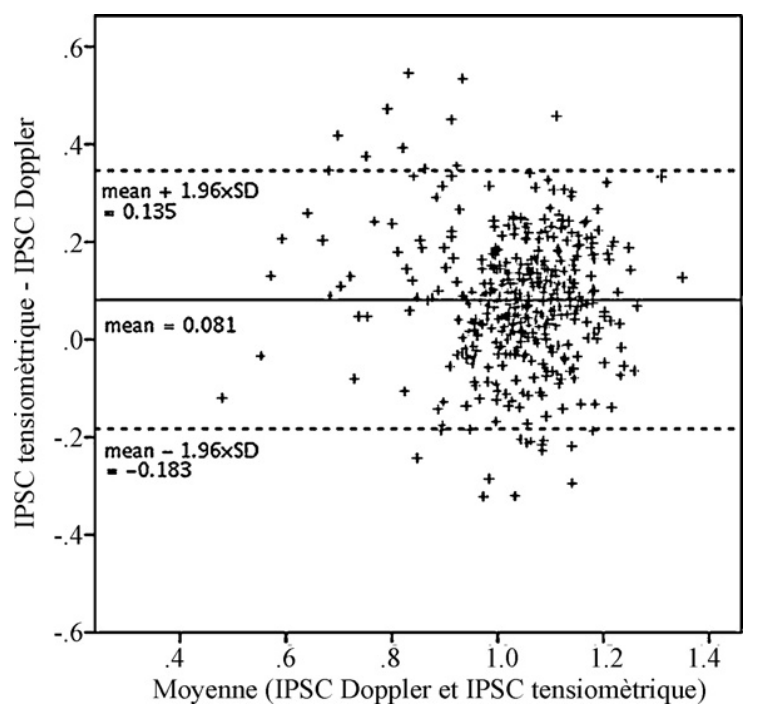

Figure 3 Représentation de Brand-Altman pour la reproductibilité entre les méthodes tensiométrique et Doppler. On retrouve en abscisse la moyenne des deux mesures et en ordonnée la différence qui les sépare.

Brand-Altman plot of reproducibility between the automatic oscillometric method and the Doppler method. The means for the two methods are presented on the $x$-axis and the difference between the methods on the $y$-axis. 
d'où un Youden index à 0,31 , une valeur prédictive positive de $59 \pm 6 \%$ et une valeur prédictive négative de $91 \pm 4 \%$.

\section{Discussion}

L'usage du doppler dans la détermination de l'IPSC a été décrit pour la première fois par Carter en 1968 [37]. Le doppler continu est depuis devenu la méthode de référence dans la mesure de l'IPSC et, par suite, dans le diagnostic d'AOMI.

Cependant cette méthode n'est pas largement employée par les praticiens en dépit de la recommandation de dépister systématiquement l'AOMl chez les patients de 70 ans et plus. L'emploi de cette méthode requiert, en effet, un équipement spécialisé et une préparation du patient d'environ 15 minutes. Une étude portant sur des patients de médecine générale aux États-Unis a constaté que plus de la moitié des patients souffrant d'AOMI avaient été diagnostiqués lors de leur admission [38], ce qui illustre le nombre important de patients artériopathes non diagnostiqués dans la population générale. Dans notre étude où la population source a été choisie pour correspondre à celle nécessitant un dépistage selon les critères contenus dans les American College of Cardiology-American Heart Association (ACC/AHA) practice guidelines [31], on a retrouvé une AOMI chez un patient sur quatre.

Une technique plus simple et plus rapide faciliterait le diagnostic de l'AOMI en ambulatoire en augmentant le nombre de dépistages réalisés.

La mesure de pression sanguine par tensiomètre automatique est apparue en pratique clinique en 1976, cependant son usage dans la mesure de l'IPSC n'a pas été clairement validé en dépit des avantages pratiques que cette méthode pourrait procurer [35].

Notre étude comporte un biais de mesure: toutes les mesures ont été réalisées par le même examinateur afin que les résultats ne soient pas opérateur-dépendants. Il ne nous est donc pas possible de mesurer la concordance interobservateur. Cependant, l'influence de l'opérateur sur la mesure de l'IPSC par la méthode Doppler est connue comme très importante $[39,40]$. Par ailleurs, nous n'avons utilisé qu'un seul type de brassard automatique, largement utilisé en France. Aussi, il convient d'être prudent pour extrapoler ces résultats à d'autres appareils disponibles sur le marché.

Notre étude a montré une mauvaise concordance entre l'indice obtenu par Doppler et par méthode par tensiomètre automatique. La variabilité des résultats est suffisamment importante pour perturber les décisions cliniques. L'intervalle de confiance à $95 \%([-0,18-0,35])$ des différences entre les résultats des deux méthodes est trop large pour être acceptable en pratique clinique. Par exemple, chez un sujet ayant un IPSC Doppler à 1,00, une mesure par tensiomètre automatique pourrait donner des valeurs allant de 0,82 à 1,35 .

Plusieurs études antérieures ont utilisé le coefficient de corrélation de Pearson entre la méthode de référence et celle par tensiomètre automatique ainsi que les tracés obtenus selon la méthode de Bland et Altman; cependant ces données n'étaient pas interprétées. Après analyse, les données de ces études révèlent, conformément aux résultats de notre étude, une importante variabilité dans les résultats obtenus par la méthode utilisant le tensiomètre automatique.

Comparativement à la méthode de référence Doppler, la détermination d'un IPSC par tensiomètre automatique ne permet pas un dépistage fiable d'une $A O M I$ en raison de son manque de sensibilité et de sa mauvaise validité prédictive.

\section{Conclusion}

Au vu des résultats obtenus par cette étude réalisée chez des patients ne souffrant pas d'artériopathie des membres inférieurs connue lors de l'inclusion et candidats au dépistage de cette pathologie, il ressort que les mesures d'IPSC obtenues par méthode tensiométrique automatique sont peu fiables. Cette méthode ne peut donc être considérée comme une alternative à la méthode Doppler, malgré les avantages pratiques qu'elle peut apporter.

La méthode de référence de détermination de I'IPSC reste donc le doppler continu. Aussi, la formation pratique de praticiens à la mesure de l'IPSC par cette méthode est une question essentielle de santé publique.

\section{Conflit d'intérêt}

Aucun.

\section{Références}

[1] Yusuf S, Reddy S, Ounpuu S, Anand S. Global burden of cardiovascular diseases: part I: general considerations, the epidemiologic transition, risk factors, and impact of urbanization. Circulation 2001;104:2746-53.

[2] Yusuf S, Reddy S, Ounpuu S, Anand S. Global burden of cardiovascular diseases: part II: variations in cardiovascular disease by specific ethnic groups and geographic regions and prevention strategies. Circulation 2001;104:2855-64.

[3] Pasternak RC, Criqui MH, Benjamin EJ, Fowkes FG, Isselbacher EM, McCullough PA, et al. AHA conference proceedings. Atherosclerotic Vascular Disease Conference. Writing group I: Epidemiology. Circulation 2004;109:2605-12.

[4] Newman AB, Siscovick DS, Manolio TA, Polak J, Fried LP, Borhani NO, et al. Ankle-arm index as a marker of atherosclerosis in the Cardiovascular Health Study. Circulation 1993;88:837-45.

[5] Abbott RD, Rodriguez BL, Petrovitch H, Yano K, Schatz IJ, Popper JS, et al. Ankle-brachial blood pressure in elderly men and the risk of stroke: the Honolulu Heart Program. J Clin Epidemiol 2001;54:973-8.

[6] Aboyans V, Criqui MH. Can we improve the cardiovascular risk prediction beyond risk equations in the physician's office? J Clin Epidemiol 2006;59:547-58.

[7] Doobay AV, Anand SS. Sensitivity and specificity of the ankle-brachial index to predict future cardiovascular outcomes: a systematic review. Arterioscler Thromb Vasc Biol 2005;25:1463-9.

[8] Belch JJF, Topol EJ, Agnelli G, Bertrand M, Califf RM, Clement $\mathrm{DL}$, et al. Critical issues in peripheral arterial disease detection and management: a call to action. Arch Intern Med 2003;163:884-92.

[9] AHA Prevention Conference V, Writing Group III. Beyond secondary prevention: identifying the high-risk patient for primary prevention. Non invasive tests of atherosclerotic burden. Circulation 2000;286:1317-24. 
[10] Norgren L, Hiatt WR, Dormandy JA, Nehler MR, Harris KA, Fowkes FG, et al. Inter-society consensus for the management of peripheral arterial disease (TASC II). Eur J Vasc Endovasc Surg 2007;33:S1-75.

[11] McDermott MM, Criqui MH, Liu K, Guralnik JM, Greenland P, Martin GJ, et al. Lower ankle/brachial index, as calculated by averaging the dorsalis pedis and posterior tibial arterial pressures, and association with leg functioning in peripheral arterial disease. J Vasc Surg 2000;32:1164-71.

[12] Lundin $M$, Wiksten JP, Peräkylä T, Lindfors $O$, Savolainen $H$, Skyttä J, et al. Distal pulse palpation: is it reliable? World J Surg 1999;23:252-5.

[13] Criqui MH, Fronek A, Klauber MR, Barrett-Connor E, Gabriel $\mathrm{S}$. The sensitivity, specificity, and predictive value of traditional clinical evaluation of peripheral arterial disease; results from non invasive testing in a defined population. Circulation 1985;71:516-22.

[14] Beckman JA, Higgins CO, Gerhard-Herman M. Automated Oscillometric Determination of the Ankle-Brachial Index provides accuracy necessary for office practice. Hypertension 2006;47:35-8.

[15] Mohler ER, Treat-Jacobson D, Reilly MP, Cunningham KE, Miani $M$, Criqui $M H$, et al. Utility and barriers to performance of the ankle-brachial index in primary care practice. Vasc Med 2004;9:253-60.

[16] Simon A, Papoz L, Ponton A, Segond P, Becker F, Drouet L, et al. Feasibility and reliability of ankle-arm blood pressure index in preventive medicine. Angiology 2000;51:463-9.

[17] Pessenhofer $H$. Single cuff comparison of two methods for indirect measurement of arterial blood pressure: standard auscultatory methods versus automatic oscillometric method. Basic Res Cardiol 1986;81:101-9.

[18] Imai Y, Abe K, Saski S, Minami N, Munakata M, Sakuma H, et al. Clinical evaluation of semiautomatic and automatic devices for home blood pressure measurement: comparison between cuff-oscillometric and microphone methods. J Hypertens 1989;7:983-90.

[19] Adiseshiah M, Cross FW, Belsham PA. Ankle blood pressure measured by automatic oscillotonometry: a comparison with Doppler pressure measurements. Ann R Coll Surg Engl 1987;69(2):71-273.

[20] Lee BY, Campbell JS, Berkowitz P. The correlation of ankle oscillometric blood pressures and segmental pulse volumes to Doppler systolic pressures in arterial occlusive disease. J Vasc Surg 1996;23:116-22.

[21] Ramanathan A, Conaghan PJ, Jenkinson AD, Bishop CR. Comparison of ankle-brachial pressure index measurements using an automated oscillometric device with the standard Doppler ultrasound technique. ANZ J Surg 2003;73:105-8.

[22] Benchimol A, Bernard V, Pillois X, Hong NT, Benchimol D, Bonnet $\mathrm{J}$. Validation of a new method of detecting peripheral artery disease by determination of ankle-brachial index using an automatic blood pressure device. Angiology 2004;55:127-34.

[23] Pan CR, Staessen JA, Li Y, Wang JG. Comparison of three measures of the ankle-brachial blood pressure index in a general population. Hypertens Res 2007;30:555-61.

[24] MacDonald E, Froggatt P, Lawrence G, Blair S. Are automated blood pressure monitors accurate enough to calculate the ankle brachial pressure index? J Clin Monit Comput 2008;22: $381-4$.
[25] MacDougall AM, Tandon V, Wilson MP, Wilson TW. Oscillometric measurement of ankle-brachial index. Can J Cardiol 2008;24:49-51.

[26] Diehm N, Dick F, Czuprin C, Lawall H, Baumgartner I, Diehm C. Oscillometric measurement of ankle-brachial index in patients with suspected peripheral disease: comparison with Doppler method. Swiss Med Wkly 2009;139:357-63.

[27] Altman DG, Bland JM. Measurement in medicine: the analysis of method comparison studies. The Statistician 1983;32:307-17.

[28] Bland JM, Altman DG. Statistical methods for assessing agreement between two methods of clinical measurement. Lancet 1986;1:307-10.

[29] Bartko JJ. The intraclass correlation coefficient as a measure of reliability. Psychol Reports 1966;19:3-11.

[30] Vinyoles E, Pujol E, Casermeiro J, de Prado C, Jabalera S, Salido V. Ankle-brachial index to detect peripheral arterial disease: concordance and validation study between Doppler and an oscillometric device. Med Clin (Barc) 2007;128:92-4.

[31] Hirsch AT, Haskal ZJ Hertzer NR, Bakal CW, Creager MA, Halperin JL et al. ACC/AHA 2005 guidelines for the management of patients with peripheral arterial disease: a collaborative report. J Am Coll Cardiol 2006;47:1239-312. Available at: http://www.acc.org/clinical/guidelines/pad/index.pdf.

[32] Anastas ZM, Jimerson E, Garolis S. Comparison of noninvasive blood pressure measurements in patients with atrial fibrillation. J Cardiovasc Nurs 2008;23:519-24.

[33] Working Group on Blood Pressure Monitoring of the European Society of Hypertension International. Blood Press Monit 2003;8:17-8.

[34] Topouchian JA, El Assaad MA, Orobinskaia LV, El Feghali RN, Asmar RG. Validation of two automatic devices for selfmeasurement of blood pressure according to the international protocol of the European society of hypertension: the Omron M6 (HEM-7001-E) and the Omron R7 (HEM 637-IT). Blood Press Monit 2006;11:165-71.

[35] Rouquette A, Tomas J, Leborgne C, Dagorne C, Fraguin F, Fernandez MS, Fanello $S$. Intérêt du calcul de l'index de pression systolique à la cheville (IPSC) par tensiomètre automatique dans le dépistage de l'artériopathie oblitérante des membres inférieurs (AOMI) et analyse des facteurs de risque. Angeiologie 2007;59:18-22.

[36] Grenier B, Dubreuil M, Journois D. Comparaison de deux méthodes de mesure d'une même grandeur: méthode de Bland et Altman. Ann Fr Anesth Reanim 2000;19:128-35.

[37] Carter SA. Indirect systolic pressures and pulse waves in arterial occlusive disease of the lower extremities. Circulation 1968;37:624-37.

[38] Hirsch AT, Halverson SL, Treat-Jacobson D, Hotvedt PS, Lunzer MM, Jrook $S$, et al. Peripheral arterial disease detection, awareness and treatment in primary care. JAMA 2001;286:1317-24.

[39] Aboyans V, Lacroix P, Lebourdon A, Preux PM, Ferrières J, Laskar M. The intra- and interobserver variability of ankle-arm blood pressure index according to its mode of calculation. $\mathrm{J} \mathrm{Cli}$ Epidemiol 2003;56:215-20.

[40] Aboyans V, Lacroix P, Doucet S, Preux PM, Criqui MH, Laskar $M$. Diagnosis of peripheral arterial disease in general practice: can the ankle-brachial index be measured either by pulse palpation or an automatic blood pressure device. Int J Clin Pract 2008;2:1001-7. 